\title{
Distributed Optimization for Control and Estimation
}

\section{Shervin Parvini Ahmadi}

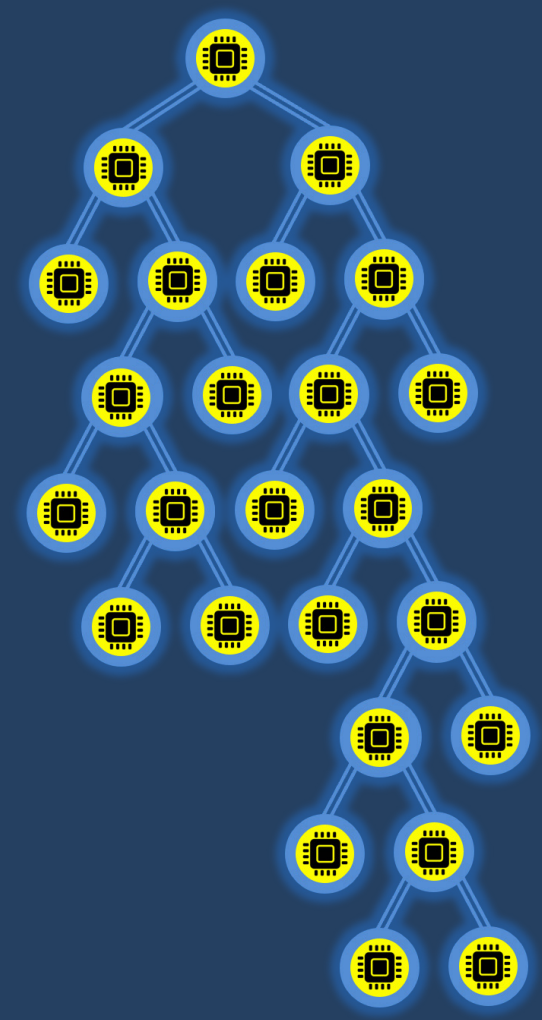



Linköping studies in science and technology. Dissertations.

No. 2207

\section{Distributed Optimization for Control and Estimation}

Shervin Parvini Ahmadi

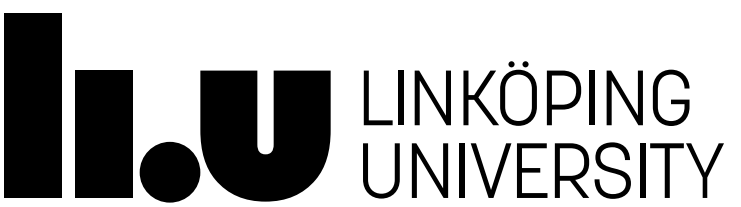


Cover illustration: A computational graph for solving an optimization problem in a distributed manner using message passing.

Linköping studies in science and technology. Dissertations.

No. 2207

Distributed Optimization for Control and Estimation

Shervin Parvini Ahmadi

shervin.parvini.ahmadi@liu.se

www. control.isy.liu.se

Division of Automatic Control

Department of Electrical Engineering

Linköping University

SE-581 83 Linköping

Sweden

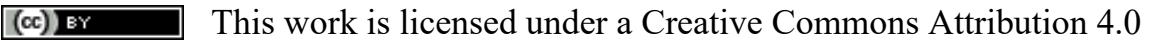
International License.

https://creativecommons.org/licenses/by/4.0/

ISBN 978-91-7929-197-6 (Print)

ISBN 978-91-7929-198-3 (PDF)ＩSSN 0345-7524

Copyright (C) 2022 Shervin Parvini Ahmadi

Printed by LiU-Tryck, Linköping, Sweden 2022 



\section{Abstract}

Adopting centralized optimization approaches in order to solve optimization problem arising from analyzing large-scale systems, requires a powerful computational unit. Such units, however, do not always exist. In addition, it is not always possible to form the optimization problem in a centralized manner due to structural constraints or privacy requirements. A possible solution in these cases is to use distributed optimization approaches. Many large-scale systems have inherent structures which can be exploited to develop scalable optimization approaches. In this thesis, chordal graph properties are used in order to design tailored distributed optimization approaches for applications in control and estimation, and especially for model predictive control and localization problems. The first contribution concerns a distributed primal-dual interior-point algorithm for which it is investigated how parallelism can be exploited. In particular, it is shown how the computations of the algorithm can be distributed on different processors so that they can be run in parallel. As a result, the algorithm execution time is accelerated compared to the case where the algorithm is run on a single processor. Simulation studies on linear model predictive control and robust model predictive control confirm the efficiency of the framework. The second contribution is to devise a tailored distributed algorithm for nonlinear least squares with application to a sensor network location problem. It relies on the Levenberg-Marquardt algorithm, in which the computations are distributed using message passing over the computational graph of the problem, which is obtained from what is known as the clique tree of the problem. The results indicate that the algorithm provides not only a good localization accuracy, but also it requires fewer iterations and communications between computational agents in order to converge compared to known first-order methods. The third contribution is a study of extending the message passing idea in order to design tailored distributed algorithm for general non-convex problems. The framework relies on an augmented Lagrangian algorithm in which a primal-dual interior-point method is used for the inner iteration. Application of the framework for general model predictive control of systems with several interconnected sub-systems is extensively investigated. The performance of the framework is then compared with distributed methods based on the alternating direction method of multipliers, where the superiority of the framework is illustrated. 



\section{Populärvetenskaplig sammanfattning}

Datorstödda beräkningar har öppnat upp för tillämpnar av optimering inom många olika ingenjörsdiscipliner. Optimal styrning och reglering av elektriska och mekaniska system och kemiska processer kan lösas med hjälp av optimering. Huvudsakligen används centraliserad optimering för detta ändamål. Detta kräver att all information om det som ska styras och regleras liksom alla beräkningar kan samlas på ett ställe. Detta är dock en utmaning för komplexa och storskaliga system, som har blivit allt vanligare de senaste åren. Speciellt kan det finnas begränsningar i centraliserad beräkningskraft, i bandbredd för kommunikation och i konfidentialitet rörande data. För att överbrygga dessa problem har distribuerad optimering föreslagits som en lösning. Inom distribuerad optimering samarbetar ett antal beräkningsenheter med varandra för att lösa ett övergripande optimeringsproblem genom att varje beräkningsenhet löser enskilda delproblem och kommunicerar med vissa av de andra beräkningsenheterna. Målet med denna avhandling är att ta fram effektiva distribuerade optimeringsmetoder för komplexa och storskaliga problem. De tillämpningar som betraktas är bl.a. modellprediktiv reglering, som är en reglermetod speciellt lämpad för att styra system med begränsningar. En annan tillämpning som studeras är lokalisering av sensorer i ett nätverk baserat på avståndsmätningar. De distribuerade metoderna vi tar fram utnyttjar inneboende strukturer i problemen vi studerar för att ta fram effektiva och resurssnåla metoder. 



\section{Acknowledgments}

I would like to start by expressing my deepest respect and gratitude to my supervisor, Anders Hansson, for the opportunity you gave me to pursue my $\mathrm{PhD}$, for your excellent guidance, continuous support, and encouragement throughout this journey. I am sincerely grateful for the positive impact you had in this chapter of my life, for your inspiring ideas, positive attitude, resourcefulness, and of course your patience! Thank you Anders! I would also like to thank Anders Helmersson and Daniel Axehill, my former and current co-supervisors, for your support and assistance.

I am very proud to be part of the group established by Lennart Ljung. I would like to deliver my gratitude to Svante Gunnarsson and Martin Enqvist, the former and current head of the division at Automatic Control, for the great leadership, maintaining an excellent working environment and in particular their invaluable kindness. A special thanks to you, Martin Enqvist for your great support towards the end of my PhD. Thanks also to Ninna Stensgård for taking care of administrative tasks and always helping me out with a smile.

This work was supported by the Wallenberg AI, Autonomous Systems and Software Program (WASP) funded by the Knut and Alice Wallenberg Foundation. My special thanks go to the people who arranged all the courses, workshops, conferences and international study trips. Their funding is gratefully acknowledged.

It is a pleasure that I got to know all my colleagues, both current and former, at Automatic Control; you made the environment very friendly. Sina Khoshfetrat Pakazad, thank you for your guidance and support at the beginning of my research. Erik Hedberg, I was very fortunate to have you around when things were not going my way. Thanks for always being there for me when I needed a listening ear, for answering all my questions and for all the inspiring discussions. Hamed Haghshenas, to have you in my corner unconditionally was very heartwarming. This indeed is very valuable for me. Thank you, Gustav Lindmark and Alberto Zenere, my former office mates, for your patience, company and all the interesting discussions. Warm thanks to Fredrik Ljungberg, Hamed Haghshenas and Pouya Mahdavipour for proofreading this thesis, your comments and time. Thank you also Gustaf Hendeby for providing the template used to write this thesis and also for all the help you have given me throughout these years. It is greatly appreciated. Alberto Zenere, Magnus Malmström and Fredrik Ljungberg, I really liked our intimate small group. The times we shared together, from the Sunday fikas to our chess, frisbee, paddle tennis events are very memorable for me. Filipe Barbosa and Jonas Nordlöf, although I got to know you at later stages of my PhD journey, we quickly became good friends and I really enjoyed the time we spent in our lunch discussions, kayak trip and board game evenings. Sara Svensson, thank you for all the amazing activities, from arranging the chocolate ball competition, crayfish party, Swedish midsummer celebrations (with Magnus as the co-organizer) to welcoming us to your hometown, Hässleholm. Thank you also Lucas Egidio (together 
with Fernanda) for all the wonderful evenings, in particular for my favorite Festa Junina, and also for your warm welcome in Brussels. Kamiar Radnosrati, Mahdieh Sadabadi, Angela Fontan, Parinaz Kasebzadeh, Du Ho, Emina Alickovic, Kristoffer Bergman, Per Bostrm-Rost, Farnaz Adib Yaghmaie and everyone else, thank you all for the great times we shared from the dinners, parties to our planning meetings.

I also would like to thank my friends outside of the Automatic Control group, who made my time more enjoyable in Sweden. Pouya Mahdavipour, Ahad Hamednia and Behnam Nouri, the closest ones to me in Sweden! The ones that I can always trust given our longtime friendship. Fatemeh Mohseni, your friendship has been an invaluable gift during the last years. Thanks for the great times we shared and for all the memorable trips and adventures we had, from exploring the Eiffel Tower, eating pasta in Venice to hiking the Great Wall of China. I started my Sweden journey together with Iman Shafikhani and shared flats for a couple of years. I am very grateful for your brotherly attitude and for your support in my difficult times. I will always remember the good times we shared, from our amazing cruise trip in the Baltic sea, exploring the Roman Colosseum, walking in Florence, to our great Jujeh Kabab BBQs. Sandra Knopik, the best Brazilian ever, I am very happy to have known you, with that never-ending happy smile and being up for every kind of adventure from amazing Brazilian parties to exploring Sweden from the west coast to Gotland. I am very fortunate to have had my wonderful friends Roghayeh Hajizadeh, Fatemeh Mohseni, Mobin Hajmohammadi, Nasim Bioukaghazadeh, Hamed Haghshenas, Shamisa Shoja and Narges Mohammadi, who always made me feel as I am home. Thanks for the great times we have shared and also for keeping our Iranian traditions and celebrations alive. Special thanks to Roghayeh Hajizadeh for helping me whenever I needed it. I want to also mention Sergii Voronov, Jana Bosca, Mahin Kiwi, Hamed Sadeghi, Mahdi Morsali and Shima Dopeikar, thanks for the nice times and good memories we have shared during the past years.

Old friends are gold! Pouya Mahdavipour, Behzad Jabbari, Sina Heidari, Mirsalim Ghoddousifar, Shahin Nokhbeh, Ahad Hamednia, Afshar Bagheri, Naser Hassanpour, Amin Ashraf Gandomi, Rasa Rikhtegaran, Aydin Mashroutechi, Faraz Zarghami, Armin Rasoulipour, Farshad Rafiei, Amin Zamani, Arash Pourmoazzen, Masood Imani, Siyamak Kaviyan, Behnam Nouri, Yashar Rafati, Yasser Mohammadi Qaragoez, Farzad Mehrpour, Sajad Naderi Lordejani and Kanan Sehat, our friendships are timeless and the memories we have created together are priceless for me. Although we are spread out all over the world and cannot see each other as often as before, it does not change the fact that you will always have special places in my heart. I look forward to seeing you again more often in the future.

Last but by no means least, my family! My father Khalil (Naghi) and my mother Nazila, I am truly blessed to have you in my life. Thanks for your endless love, encouragement, your unconditional support all the way to this point and for always believing in me. My sister Sara and my brother-in-law Omid, thanks for 
always being there for me and for welcoming me so warmly every time I visit you in my birthplace, Strasbourg. I love you all.

Linköping, January 2022 Shervin Parvini Ahmadi 



\section{Contents}

\section{Background}

1 Introduction $\quad 3$

1.1 Contributions . . . . . . . . . . . . . . . . 6

1.2 Thesis Outline and Publications . . . . . . . . . . . . . 6

1.3 Background . . . . . . . . . . . . . . . . . 10

1.3.1 Coupled Optimization Problems . . . . . . . . . . . 10

1.3.2 Coupling and Sparsity Graphs . . . . . . . . . . . . 10

1.3.3 Chordal Graphs and Clique Trees . . . . . . . . . . . 11

1.3.4 Optimization over Clique Trees Using Message Passing . . . 15

2 Concluding Remarks and Future Work $\quad 19$

$\begin{array}{ll}\text { Bibliography } & 21\end{array}$

\section{Publications}

A Parallel Exploitation for Tree-Structured Coupled Quadratic Program$\begin{array}{lr}\text { ming in Julia } & \mathbf{2 9}\end{array}$

1 Introduction . . . . . . . . . . . . . . . . . . 31

2 Simple Example . . . . . . . . . . . . . . . . . 33

3 Problem Formulation . . . . . . . . . . . . . . . . . 35

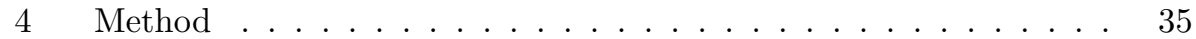

5 Julia Implementation . . . . . . . . . . . . . . . . . . . . . . . . . . . . . . . . . . . 36

6 Numerical Experiments . . . . . . . . . . . . . . . . . . . . . 37

$6.1 \quad$ Scenario $1 \ldots \ldots \ldots 38$

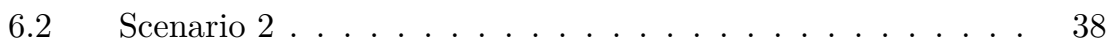

6.3 Scenario $3 \ldots \ldots \ldots 40 \ldots \ldots$

7 Conclusion ........................... 41

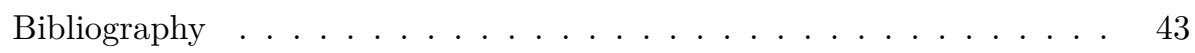


B Efficient Robust Model Predictive Control Using Chordality 45

1 Introduction . . . . . . . . . . . . . . . . . . . . . . . 47

2 Robust MPC . . . . . . . . . . . . . . . . . . . 48

3 Robust Quadratic Programming . . . . . . . . . . . . . 50

4 Chordal Sparsity and Convex Optimization . . . . . . . . . . 51

5 Implementation . . . . . . . . . . . . . . . . . . 55

6 Numerical Experiments . . . . . . . . . . . . . . . . . . . 55

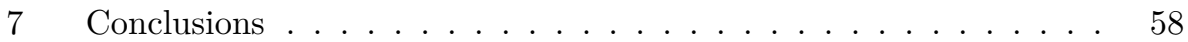

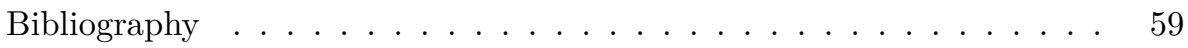

C Distributed Localization Using Levenberg-Marquardt Algorithm $\quad 63$

1 Introduction . . . . . . . . . . . . . . . . 65

2 Maximum Likelihood Localization _. . . . . . . . . . . . . . . . . . 72

3 Clique Tree and Assignment Strategy . . . . . . . . . . . . . . 73

4 Levenberg-Marquardt Algorithm . . . . . . . . . . . . . . . . . . 75

5 Distributed Computations . . . . . . . . . . . . . . . 78

6 Results and Discussion . . . . . . . . . . . . . . . . . . 80

$6.1 \quad$ Simulation Data . . . . . . . . . . . . . . 81

6.2 Performance Assessment . . . . . . . . . . . . . . . . 81

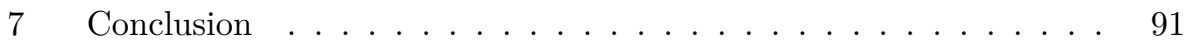

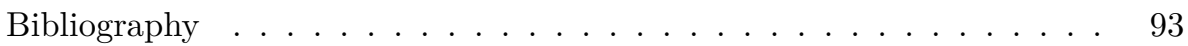

D A Distributed Second-Order Augmented Lagrangian Method for Dis$\begin{array}{ll}\text { tributed Model Predictive Control } & 97\end{array}$

1 Introduction . . . . . . . . . . . . . . . . . . . . . 99

2 Augmented Lagrangian Formulation . . . . . . . . . . . . . . . . . 102

3 Distributed Computation for the Inner Iteration . . . . . . . . . . 104

4 Numerical Experiments . . . . . . . . . . . . . . 106

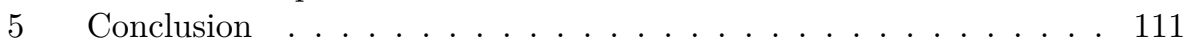

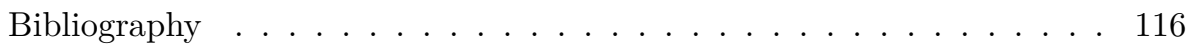

E Distributed Model Predictive Control Using an Augmented Lagrangian $\begin{array}{lr}\text { Method } & \mathbf{1 1 9}\end{array}$

1 Introduction . . . . . . . . . . . . . . . . . . . 121

2 Augmented Lagrangian and Primal-Dual Interior-Point Methods . 124

3 Distributed Computation for the Inner Iteration . . . . . . . . . . 130

4 Numerical Experiments . . . . . . . . . . . . . . . . . . . . 134

4.1 Randomly Generated Interconnected Sub-Systems . . . . . 134

4.2 Vehicle Platooning Problem . . . . . . . . . . . . . . 139

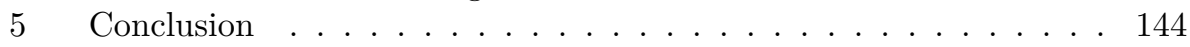

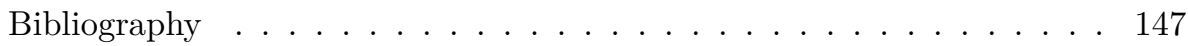


Part I

\section{Background}





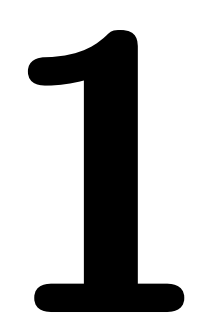

\section{Introduction}

There has been a considerable interest in distributed optimization over the recent years due to the advances in technology. Distributed optimization is the procedure of solving an optimization problem using a network of computational agents. Particularly, the complete problem is decomposed into several local problems, which are assigned to the computational agents. The agents subsequently solve the complete optimization problem by collaborating and communicating with each other. The special case where only one computation agent is used, is called centralized optimization. This has been the primary way to solve optimization problems in the past. There are, however, a number of limitations associated with centralized optimization. The first one is the computational challenges, particularly memory and power insufficieny, (Boyd et al., 2011). These challenges arise when analyzing large-scale complex systems with a substantial number of decision variables. The second limitation is the communication challenges. Recent advances in wired and wireless technology have led to the appearance of large-scale networked systems. These systems comprise a significant number of interconnected sub-systems which cooperate with each other to achieve a desired objective. Therefore, using a centralized optimization approach will require a considerable number of communications due to the distributed nature of them which is not desired, (Yang et al., 2019). The third limitation is the incapability of the centralized optimization approaches in handling applications with structural constraints. For instance, if there is a requirement on preserving the privacy of data, then it is not always possible to form the optimization problem in a centralized manner, (Nedić and Liu, 2018; Pakazad, 2015; Zheng, 2019). In order to address these limitations, distributed optimization approaches have extensively been studied over the past years. Two pioneer early works are Bertsekas and Tsitsiklis (1997); Tsitsiklis (1984). The applications of distributed optimization in control and estimation are very broad, e.g., wireless sensor networks, (Biswas et al., 2006; Simonetto and Leus, 2014; Wang et al., 2008), power systems, (Kargarian et al., 2016; Molzahn et al., 2017), autonomous 
vehicles, (Bian et al., 2019; Cao et al., 2012; Khayatian et al., 2020; Malikopoulos et al., 2018; Tajalli and Hajbabaie, 2018), machine learning, (Boyd et al., 2011; Nedic, 2020), robotic networks, (Bullo et al., 2008), communication networks, (Johansson, 2008; Nedić et al., 2018; Sayed, 2014), and multi-agent systems, (Fioretto et al., 2018; Ren and Cao, 2011; Zhu and Martínez, 2015), to name a few. For a thorough and recent review of distributed optimization algorithms, refer to Boyd et al. (2011); Molzahn et al. (2017); Nedić and Liu (2018); Nedich et al. (2015); Yang and Johansson (2010); Yang et al. (2019).

The classical distributed optimization approaches are based on applying firstorder methods to the problem or some formulation of the problem. First-order methods are methods which make use of gradients of the objective and constraint functions. These approaches rely on exact gradient computations if the problem is smooth, otherwise they rely on subgradients or proximal operators, (Bertsekas and Tsitsiklis, 1997; Eckstein, 1989; Nedic and Ozdaglar, 2009). In this class of distributed approaches, some popular algorithms are based on the Alternating Direction Method of Multipliers (ADMM), which is a special case of the proximal point method, (Boyd et al., 2011). Although these approaches are effective, a major shortcoming is their slow rates of convergence. In order to alleviate this issue, there has recently been an interest in devising distributed approaches based on second-order or Newton type methods, such a interior-point methods, due to their superior convergence rate. Second-order methods are methods which make use of both gradients and Hessians of the objective and constraint functions. See Dinh et al. (2013); Houska et al. (2016); Necoara and Suykens (2009), for examples of distributed second-order approaches and the references therein. In Necoara and Suykens (2009), a distributed algorithm based on a Lagrangian dual decomposition technique is presented for convex optimization problems. The dual variables are updated in an interior-point framework using the second-order information of the dual function. The algorithm is limited to problems with affine constraints, and also it requires that each agent communicates its local primal variables with all the other agents at each iteration, in order to attain the necessary information for updating the dual variables. In Dinh et al. (2013), a dual decomposition based distributed method is presented for convex problems with affine constraints, which employs an interior-point framework with a smoothed dual function. A limitation of the algorithm is that it often requires a large number of iterations in order to converge to a solution with medium accuracy, (Houska et al., 2016). In Houska et al. (2016), a distributed algorithm for non-convex smooth optimization problems with coupled affine constraints is presented. The algorithm is a further development of ADMM, which contains ideas from augmented Lagrangian and sequential quadratic programming. The algorithm has a faster convergence rate compared to ADMM based algorithms. This however comes at the price that it requires more communications, and also the centralized update cannot be distributed, (MeyerHuebner et al., 2019). Common for the distributed approaches based on second order information is that they do not address general nonlinear constraints.

In this thesis, we continue the lines of research initialized in Khoshfetrat Pakazad et al. (2017), for distributed optimization of loosely coupled convex problems. The algorithm is a the primal-dual interior-point method and enjoys superior conver- 
gence properties compared to other existing distributed algorithms. We extend the applicability to non-convex smooth optimization problems with general nonlinear constraints. The algorithm is based on an augmented Lagrangian formulation. As we will see later, all the computations of the algorithm can be distributed, and also the required communications for convergence is much lower than for distributed ADMM based methods.

Most optimization problems arising in the aforementioned applications have inherent structures and are sparse in their topological connections. The main motivation of this thesis is to exploit the sparsity in these optimization problems. A common structure which is exploited in devising a majority of the distributed algorithms is what is known as partial separability. A partial separable function can be defined as a function which can be defined as summation of elementary functions, where each elementary function depends on a small subset of the variables, (Vandenberghe and Andersen, 2015). A more precise definition will be provided later. Examples of where partial separability is exploited within optimization algorithms include the distributed approaches based on the Douglas-Rachford method in Parikh and Boyd (2014); Spingarn (1985), the ADMM in Boyd et al. (2011), the quasi-Newton method in Griewank and Toint (1982a,b); (Nocedal and Wright, 2006, Ch. 7), the trust-region method in Colson and Toint (2005); Conn et al. (2000), the proximal method in Chen and Teboulle (1994); Tseng (1991), the dual block coordinate ascent method in Tseng (1993), and the interior-point method in Gondzio and Sarkissian (2003); Kojima et al. (1994). Also, the nonlinear optimization software, LANCELOT, (Conn et al., 1992), exploits partial separability. Several of the algorithms that exploit partial separability do this by exploiting so-called chordal properties; see Vandenberghe and Andersen (2015) and the references therein. Chordal graphs are well-studied in graph theory and have several applications in different fields. One well-known application is to solve sparse linear systems via Cholesky factorization, (Zheng, 2019). Also, there are fundamental properties that are shared between chordal graphs and sparse positive semidefinite matrices. These properties are extensively used in order to solve large semidefinite optimization problems, (Vandenberghe and Andersen, 2015). One other important application is in probabilistic inference and machine learning where chordal graphs are exploited in solving maximum likelihood estimation problems, (Koller and Friedman, 2009; Wainwright and Jordan, 2008). In this thesis, chordal graphs are used in order to design distributed optimization algorithms, tailored for two different applications, namely the Model Predictive Control (MPC) problem and the problem of sensor network localization. For the MPC application, different type of problems are considered. In particular, linear MPC and robust MPC problems are investigated, and a distributed primal-dual interior-point method is presented in which one can exploit parallelism. It should be noted that a main application of distributed optimization is in parallel implementation using multiple processors. This, in turn, accelerates the execution time of the algorithm compared to the case where the algorithm is run in a single processor. Nonlinear MPC problem is also explored for a network of inter-connected sub-systems and a distributed algorithm is devised based on the augmented Lagrangian and a primal-dual interior-point method. As for the sensor network localization problem, a distributed algorithm 
is proposed which relies on the Levenberg-Marquardt method. In order to distribute the computations within each of the above-mentioned algorithms, the idea of message passing over a computational graph, known as a clique tree, is adopted from Khoshfetrat Pakazad et al. (2017). This can also be interpreted as doing dynamic programming over a tree, (Bertele and Brioschi, 1973).

\subsection{Contributions}

The main contributions of the thesis can be summarized as: (I) Parallel exploitation for a distributed primal-dual interior-point algorithm. (II) Development of a distributed Levenberg-Marquardt based algorithm for nonlinear least squares problems. (III) Development of a distributed augmented Lagrangian based algorithm for general non-convex problems. The detailed contributions are as follows:

- Implementation of a distributed primal-dual interior-point algorithm for loosely coupled Quadratic Programming (QP) problems with affine equality and convex inequality constraints in Julia. Algorithm execution time acceleration via parallel exploitation. Application of the algorithm to linear MPC. Application of the algorithm to robust MPC via reformulating it as a Robust Quadratic Programming (RQP) problem. (Papers A and B)

- Extension of the message passing idea to a nonlinear least squares problem. Development and implementation of a distributed algorithm based on the Levenberg-Marquardt algorithm. Application of the algorithm to the sensor network localization problem. Extensive simulations in Julia and performance analysis with regard to competitive methods in the literature. (Paper C)

- Extension of the message passing idea to non-convex problems with equality and inequality constraints. Development and implementation of a distributed algorithm based on a second-order augmented Lagrangian and primaldual interior-point methods. Application of the algorithm to distributed general MPC. Performance analysis with regard to a competitive distributed method. Application of the method to a vehicle platooning problem. (Papers $\mathrm{D}$ and $\mathrm{E}$ )

\subsection{Thesis Outline and Publications}

The thesis is divided into two parts, where the first part provides the background material and the second part is a collection of the papers listed below. Here, a summary of each paper together with the contributions of the thesis author are also provided.

\section{Paper A: Parallel Exploitation for Tree-Structured Coupled Quadratic Program- ming in Julia}


Paper $\mathrm{A}$ is an edited version of

Shervin Parvini Ahmadi and Anders Hansson. Parallel exploitation for tree-structured coupled quadratic programming in Julia. In 2018 22nd International Conference on System Theory, Control and Computing (ICSTCC), pages 597-602. IEEE, 2018.

Summary: In this paper we implement a distributed primal-dual interior-point algorithm in Julia for loosely coupled QP problems. We consider affine equality and affine inequality constraints. The algorithm is based on message passing in which at each iteration we compute search direction and step size, distributedly. We exploit parallelism by running the algorithm on multiple processors. This, in turn, accelerate the execution time of the algorithm. We evaluate the performance of the algorithm on linear MPC problems.

Comment: The author of this thesis contributed with the majority of the work including implementations, numerical calculations, analysis and preparation of the manuscript.

\section{Paper B: Efficient Robust Model Predictive Control Using Chordality}

Paper B is an edited version of

Shervin Parvini Ahmadi, Anders Hansson, and Sina Khoshfetrat Pakazad. Efficient robust model predictive control using chordality. In 2019 18th European Control Conference (ECC), pages 4270-4275. IEEE, 2019.

Summary: In this paper we implement a distributed primal-dual interior-point algorithm in Julia for loosely coupled QP problems with affine equality and convex inequality constraints. We apply it on a robust MPC problem. To this end, we first reformulate the problem as a RQP problem. We then show how the chordal structure can be used to distribute the computations in the algorithm which allows us to exploit parallelism.

Comment: The author of this thesis contributed with the majority of the work including implementations, numerical calculations, analysis and preparation of the manuscript.

\section{Paper C: Distributed Localization Using Levenberg-Marquardt Algorithm}

Paper $\mathrm{C}$ is an edited version of

Shervin Parvini Ahmadi, Anders Hansson, and Sina Khoshfetrat Pakazad. Distributed localization using Levenberg-Marquardt algorithm. EURASIP Journal on Advances in Signal Processing, 2021(1):1-26, 2021.

Summary: In this paper we present a distributed algorithm for the sensor network localization problem which is formulated using maximum likelihood estimation. 
The framework is based on the Levenberg-Marquardt algorithm in which the computations are distributed using message passing over the clique tree of the problem. We show that the framework provides a good localization accuracy and requires fewer iterations and communications between computational agents in comparison to first-order methods.

Comment: The author of this thesis contributed with the majority of the work including algorithm development, implementations, numerical calculations, analysis and preparation of the manuscript.

\section{Paper D: A Distributed Second-Order Augmented Lagrangian Method for Dis- tributed Model Predictive Control}

Paper D is an edited version of

Shervin Parvini Ahmadi and Anders Hansson. A distributed secondorder augmented Lagrangian method for distributed model predictive control. IFAC-PapersOnLine, 54(6):192-199, 2021a.

Summary: In this paper we present a distributed algorithm for nonlinear MPC of systems consisting of several interconnected sub-systems. The algorithm is based on a second-order augmented Lagrangian method in which the computations are distributed using message passing over the clique tree of the problem. We show the efficiency of the algorithm in terms of iterations and communications required for convergence.

Comment: The author of this thesis contributed with the majority of the work including algorithm development, implementations, numerical calculations, analysis and preparation of the manuscript.

\section{Paper E: Distributed Model Predictive Control Using an Augmented Lagrangian Method}

Paper E is an edited version of

Shervin Parvini Ahmadi and Anders Hansson. Distributed model predictive control using an augmented Lagrangian method. Submitted for possible publication, 2021b.

Summary: In this paper we present a distributed algorithm for nonlinear MPC of systems consisting of several interconnected sub-systems. Compared to the work in Paper D, in this paper we take inequality constraints into account as well. The algorithm relies on an augmented Lagrangian formalism in which a primal-dual interior-point method is used in the inner iteration. The computations of the algorithm are distributed using message passing over the clique tree of the problem. We compare performance of the algorithm to a competitive distributed ADMM based method in terms of iterations and communications required for convergence. 
Additionally, we apply the proposed algorithm to a vehicle platooning problem and illustrate its efficiency.

Comment: The author of this thesis contributed with the majority of the work including algorithm development, implementations, numerical calculations, analysis and preparation of the manuscript. 


\subsection{Background}

In this section we discuss how we can find the computational graphs used in devising the distributed algorithms in the appended papers. Throughout this thesis, we consider coupled optimization problems and we are particularly interested in the ones which are loosely coupled. Next, we present these type of problems.

\subsubsection{Coupled Optimization Problems}

A coupled optimization problem can be formulated as optimizing a sum of $N$ objective functions, i.e.

$$
\min _{x} F_{1}(x)+\cdots+F_{N}(x),
$$

where $F_{i}: \mathbb{R}^{n} \rightarrow \mathbb{R}$ for all $i=1, \ldots, N$, and $x \in \mathbb{R}^{n}$ is the optimization variable. Notice that each $F_{i}$ can describe equality or inequality constraints using indicator functions. We assume that the problem is loosely coupled meaning that each $F_{i}$ depends only on some of the components of $x$. This type of problem is called partially separable. Let us denote the ordered set of indexes of $x$ on which $F_{i}$ depends, by $J_{i} \in \mathbb{N}_{n}$. The problem in (1.1) can then be rewritten as

$$
\min _{x} \bar{F}_{1}\left(E_{J_{1}} x\right)+\cdots+\bar{F}_{N}\left(E_{J_{N}} x\right),
$$

where $E_{J_{i}}$ is a zero-one matrix obtained from an identity matrix of order $n$ by deleting the rows indexed by $\mathbb{N}_{n} \backslash J_{i}$. The function $\bar{F}_{i}$ is lower dimensional description of $F_{i}$ such that $F_{i}(x)=\bar{F}_{i}\left(E_{J_{i}} x\right)$ for all $x \in \mathbb{R}^{n}$ and $i=1, \ldots, N$. As an example, let us consider the following optimization problem:

$$
\min _{x} F_{1}(x)+F_{2}(x)+F_{3}(x)+F_{4}(x)+F_{5}(x)+F_{6}(x)+F_{7}(x),
$$

where $x \in \mathbb{R}^{15}$ and let us assume that $J_{1}=\{1,2,5,6\}, J_{2}=\{2,3,11\}, J_{3}=$ $\{4,5,7,8,12\}, J_{4}=\{3,4,13\}, J_{5}=\{9,10\}, J_{6}=\{6,7,9,14\}$ and $J_{7}=\{8,15\}$. Writing the problem in the same format as in (1.2) results in

$$
\begin{array}{ll}
\min _{x} & \bar{F}_{1}\left(x_{1}, x_{2}, x_{5}, x_{6}\right)+\bar{F}_{2}\left(x_{2}, x_{3}, x_{11}\right)+\bar{F}_{3}\left(x_{4}, x_{5}, x_{7}, x_{8}, x_{12}\right) \\
& +\bar{F}_{4}\left(x_{3}, x_{4}, x_{13}\right)+\bar{F}_{5}\left(x_{9}, x_{10}\right)+\bar{F}_{6}\left(x_{6}, x_{7}, x_{9}, x_{14}\right)+\bar{F}_{7}\left(x_{8}, x_{15}\right) .
\end{array}
$$

We can see the coupling structure of the problem more clearly in this formulation. The coupling structure can also be described via undirected graphs which is presented next.

\subsubsection{Coupling and Sparsity Graphs}

A graph $G$ is denoted by $G(V, \mathcal{E})$, where $V=\{1, \ldots, m\}$ is the set of vertices or nodes and $\mathcal{E} \subseteq V \times V$ denotes the set of edges. The coupling structure for the problem in (1.1), can be expressed using two undirected graphs, namely a coupling 


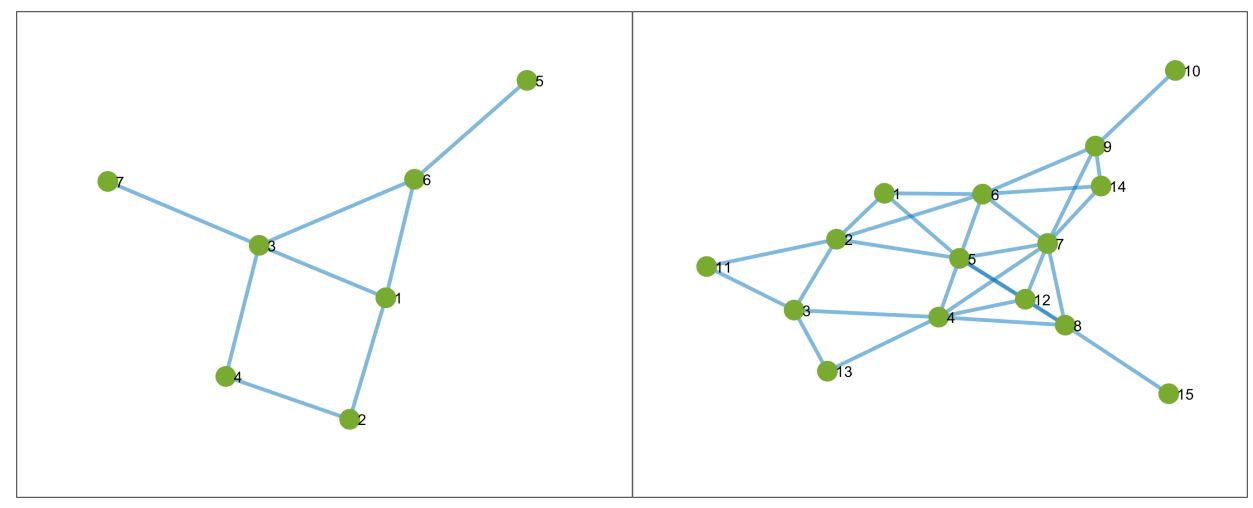

(a) Coupling graph

(b) Sparsity graph

Figure 1.1: Coupling and sparsity graphs for the problem in (1.4).

graph and a sparsity graph. The coupling graph $G_{c}$ for the problem in (1.1), is a graph with vertex set $V_{c}=\{1, \ldots, N\}$ and edge set $\mathcal{E}_{c}$ with $(i, j) \in \mathcal{E}_{c}$ if and only if $J_{i} \cap J_{j} \neq \emptyset$. The sparsity graph $G_{s}$ for the problem in (1.1), is a graph with vertex set $V_{s}=\{1, \ldots, n\}$ and edge set $\mathcal{E}_{s}$ with $(i, j) \in \mathcal{E}_{s}$ if and only if there exists an $\bar{F}_{k}$ in the equivalent formulation in (1.2) which depends on both $x_{i}$ and $x_{j}$. The coupling and sparsity graphs for the problem in (1.4) are illustrated in Figure 1.1. These graphs play a central role in designing distributed optimization methods for coupled structured problems. To be specific, depending on the coupling structure of the problem, one of these graphs will be used in order to obtain a so-called computational graph for the distributed optimization method. We will get back to this in the next section.

\subsubsection{Chordal Graphs and Clique Trees}

In order to define chordal graphs and clique trees, we first put forth a few definitions on graphs. Let us consider a graph $G(V, \mathcal{E})$ with vertex set $V$ and edge set $\mathcal{E}$. Vertices $i, j \in V$ are adjacent if $(i, j) \in \mathcal{E}$. The set of adjacent vertices or neighbors of $i$ are denoted by $\mathrm{Ne}(i)=\{j \in \mathcal{E} \mid(i, j) \in \mathcal{E}\}$. A graph is said to be complete if all its vertices are adjacent. An induced graph $Q_{I}\left(V^{\prime}, \mathcal{E}^{\prime}\right)$ on $Q(V, \mathcal{E})$, is a graph where $V^{\prime} \subseteq V$ and $\mathcal{E}^{\prime}=\mathcal{E} \cap V^{\prime} \times V^{\prime}$. A clique $C_{i}$ of $Q(V, \mathcal{E})$ is a maximal subset of $V$ that induces a complete subgraph on $Q$, i.e. no clique is properly contained in another clique, (Blair and Peyton, 1993). A sequence of vertices $\left[v_{0}, v_{1}, v_{2}, \ldots, v_{l}, v_{0}\right]$ is called a cycle of length $l+1$ if $v_{i-1}, v_{i} \in \mathcal{E}$ for $i=1, \ldots, l$ and $v_{l}, v_{0} \in \mathcal{E}$. Assume that all cycles of length at least four of $Q(V, \mathcal{E})$ have a chord, where a chord is an edge between two non-consecutive vertices in a cycle. This graph is then called chordal, (Golumbic, 2004, Ch. 4). It is possible to make a graph chordal by adding edges to the graph. This process is called triangulation and the resulting graph is referred to as a chordal embedding. Let $C_{Q}=\left\{C_{1}, \ldots, C_{q}\right\}$ denote the set of its cliques, where $q$ is the number of cliques of the graph. Then there exists a tree 


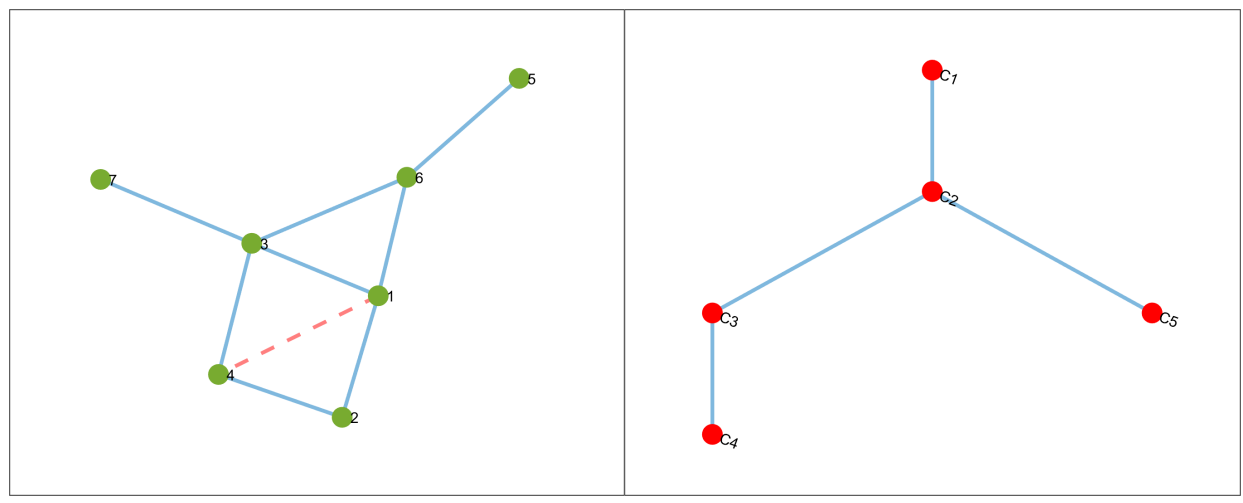

(a) Chordal embedding

(b) Clique tree

Figure 1.2: Chordal embedding of the coupling graph in Figure 1.1(a), and its corresponding clique tree

defined on $C_{Q}$ such that for every $C_{i}, C_{j} \in C_{Q}$ where $i \neq j, C_{i} \cap C_{j}$ is contained in all the cliques in the path connecting the two cliques in the tree. This property is called the clique intersection property, (Blair and Peyton, 1993). Trees with this property are referred to as clique trees.

Now as an example, let us reconsider the coupling and sparsity graphs in Figure 1.1 for the problem in (1.4). Neither of the graphs are chordal because they have cycles with a length greater than 3. For the coupling graph in Figure 1.1(a), one can simply find the chordal embedding by adding an edge between nodes 1 and 4, shown with the dashed line in Figure 1.2(a). By doing so, the corresponding cliques for this graph are

$$
\begin{aligned}
& C_{1}=\{1,2,4\}, \\
& C_{2}=\{1,3,4\}, \\
& C_{3}=\{1,3,6\}, \\
& C_{4}=\{5,6\}, \\
& C_{5}=\{3,7\} .
\end{aligned}
$$

Note that the entries of the cliques obtained from coupling graph correspond to the indexes of $\bar{F}_{i}$ in (1.4). For the sparsity graph in Figure 1.1(b), it is not trivial to find the chordal embedding by adding edges manually. There are various methods to find a chordal embedding and in particular, there are exact algorithms to find an optimal chordal embedding. These algorithms, however, are too expensive in practice. A common alternative is to use greedy search methods, which are based on different heuristic cost functions, such as min-neighbors, min-weight, min-fill, weighted-min-fill. One such method for computing the chordal embedding and its cliques is given in (Koller and Friedman, 2009, Ch. 9), which is based 
on the node-elimination technique. See also L'Excellent (2012), for a comparison between various chordal embedding methods and the resulting clique trees. Using a greedy search method in the MATLAB library Xie (2020), two different chordal embedding graphs for the sparsity graph in Figure 1.1(b), are illustrated in Figure 1.3(a) and Figure 1.4(a). The added edges are shown with the dashed lines. The corresponding cliques for the chordal embedding in Figure 1.3(a) are

$$
\begin{aligned}
& C_{1}=\{8,15\}, \\
& C_{2}=\{6,7,9,14\}, \\
& C_{3}=\{3,4,13\}, \\
& C_{4}=\{4,5,7,8,12\}, \\
& C_{5}=\{2,3,11\}, \\
& C_{6}=\{9,10\}, \\
& C_{7}=\{4,5,6,7\}, \\
& C_{8}=\{1,2,4,5,6\}, \\
& C_{9}=\{1,2,3,4\} .
\end{aligned}
$$

and for the chordal embedding in Figure 1.4(a), the corresponding cliques are

$$
\begin{aligned}
& C_{1}=\{1,2,3,5,6,11\}, \\
& C_{2}=\{1,3,4,5,6,11,13\}, \\
& C_{3}=\{4,5,6,7,9,13,14\}, \\
& C_{4}=\{4,5,7,8,9,12,13\}, \\
& C_{5}=\{4,7,8,9,10,12,13\}, \\
& C_{6}=\{8,15\} .
\end{aligned}
$$

Note that the entries of the cliques obtained from the sparsity graph correspond to the indexes of the variable $x$ in (1.4). Having computed the chordal embedding graphs and their cliques, we need to find their corresponding clique trees as well. To do so, an efficient approach is the maximum spanning tree algorithm which is presented in Koller and Friedman (2009). The corresponding clique trees for the chordal embedding graphs in Figure 1.2(a), Figure 1.3(a) and Figure 1.4(a) are illustrated in Figure 1.2(b), Figure 1.3(b) and Figure 1.4(b), respectively.

Clique trees are used as the computational graphs for designing distributed algorithms in this thesis, where each clique acts a computational agent that collaborate with its neighboring cliques. Therefore, they play an important role in the process of algorithm development. Once the clique tree is found, we choose one clique as the root of the tree. By doing so, we can define the so-called parent and children of a clique. The parent clique of clique $i$, denoted by $\operatorname{par}\left(C_{i}\right)$, is the adjacent clique of clique $i$ which is in the path connecting clique $i$ to the root clique. The children cliques of clique $i$, denoted by $\operatorname{chi}\left(C_{i}\right)$, are the adjacent cliques of clique $i$ which are not in the path connecting clique $i$ to the root clique. For 


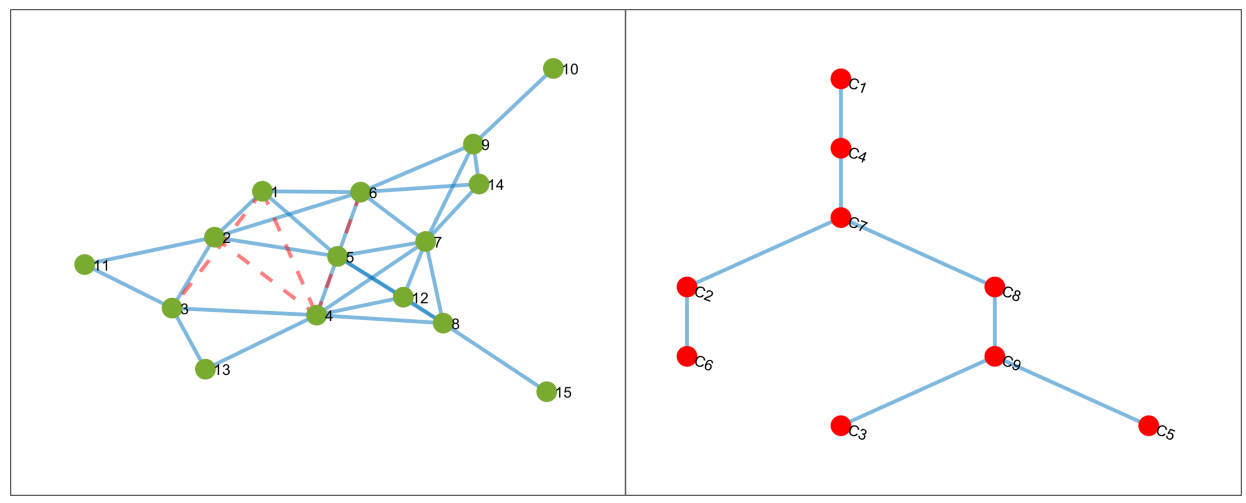

(a) Chordal embedding

(b) Clique tree

Figure 1.3: First chordal embedding of the sparsity graph in Figure 1.1(b), and its corresponding clique tree

example, for the clique tree in Figure 1.2(b), if we choose $C_{1}$ to be the root clique, then $C_{1}$ is the parent clique of $C_{2}$, and $\left\{C_{3}, C_{4}\right\}$ are the children cliques of $C_{2}$. Once the root clique is selected, we assign each $\bar{F}_{i}$ in (1.4) to a clique. We denote the ordered set of indexes of $\bar{F}_{i}$ assigned to clique $j$ by $\phi_{j}$. The assignment is not unique and can be done in different ways. Next, we describe how this assignment can be done depending on if the given graph is a sparsity graph or a coupling graph. In the case of a coupling graph, the assignment strategy is straightforward. In particular, $\bar{F}_{i}$ can only be assigned to clique $j$ if $i \in C_{j} \backslash \operatorname{par}\left(C_{j}\right)$, i.e. $i$ is an element of $C_{j}$ which in not present in $\operatorname{par}\left(C_{j}\right)$. Therefore, for the clique tree in Figure $1.2(\mathrm{~b})$, the sets $\left\{\bar{F}_{1}, \bar{F}_{2}, \bar{F}_{4}\right\},\left\{\bar{F}_{3}\right\},\left\{\bar{F}_{6}\right\},\left\{\bar{F}_{5}\right\}$ and $\left\{\bar{F}_{7}\right\}$ are assigned to cliques $C_{1}, C_{2}, C_{3}, C_{4}$ and $C_{5}$, respectively, i.e. $\phi_{1}=\{1,2,4\}, \phi_{2}=\{3\}, \phi_{3}=\{6\}$, $\phi_{4}=\{5\}$ and $\phi_{5}=\{7\}$. In the case of a sparsity graph, however, a valid assignment is that $\bar{F}_{i}$ can only be assigned to clique $j$ if $J_{i} \subseteq C_{j}$. It should be noted that there always exists a $C_{j}$ for which $J_{i} \subseteq C_{j}$, see Khoshfetrat Pakazad et al. (2017), for the proof. Therefore, for the clique tree in Figure 1.3(b), the sets $\left\{\bar{F}_{7}\right\}$, $\left\{\bar{F}_{6}\right\},\left\{\bar{F}_{4}\right\},\left\{\bar{F}_{3}\right\},\left\{\bar{F}_{2}\right\},\left\{\bar{F}_{5}\right\}$ and $\left\{\bar{F}_{1}\right\}$ are assigned to cliques $C_{1}, C_{2}, C_{3}, C_{4}$, $C_{5}, C_{6}$ and $C_{8}$, respectively, and no term is assigned to cliques $C_{7}$ and $C_{9}$. This means that $\phi_{1}=\{7\}, \phi_{2}=\{6\}, \phi_{3}=\{4\}, \phi_{4}=\{3\}, \phi_{5}=\{2\}, \phi_{6}=\{5\}, \phi_{7}=\emptyset$, $\phi_{8}=\{1\}$ and $\phi_{9}=\emptyset$. Also, for the clique tree in Figure 1.4(b), the sets $\left\{\bar{F}_{1}, \bar{F}_{2}\right\}$, $\left\{\bar{F}_{4}\right\},\left\{\bar{F}_{6}\right\},\left\{\bar{F}_{3}\right\},\left\{\bar{F}_{5}\right\}$ and $\left\{\bar{F}_{7}\right\}$ are assigned to cliques $C_{1}, C_{2}, C_{3}, C_{4}, C_{5}$ and $C_{6}$, respectively, i.e. $\phi_{1}=\{1,2\}, \phi_{2}=\{4\}, \phi_{3}=\{6\}, \phi_{4}=\{3\}, \phi_{5}=\{5\}$ and $\phi_{6}=\{7\}$.

As we stated before, either sparsity or coupling graphs can be used in order to find a computational graph for the distributed optimization method. The most commonly used one in the literature is the sparsity graph. For instance, see Khoshfetrat Pakazad et al. (2017); Vandenberghe and Andersen (2015); Wainwright and Jordan (2008). However, there are also works which use coupling graphs, for instance, see Garstka et al. (2021); Zheng et al. (2017, 2018, 2019). It should be 


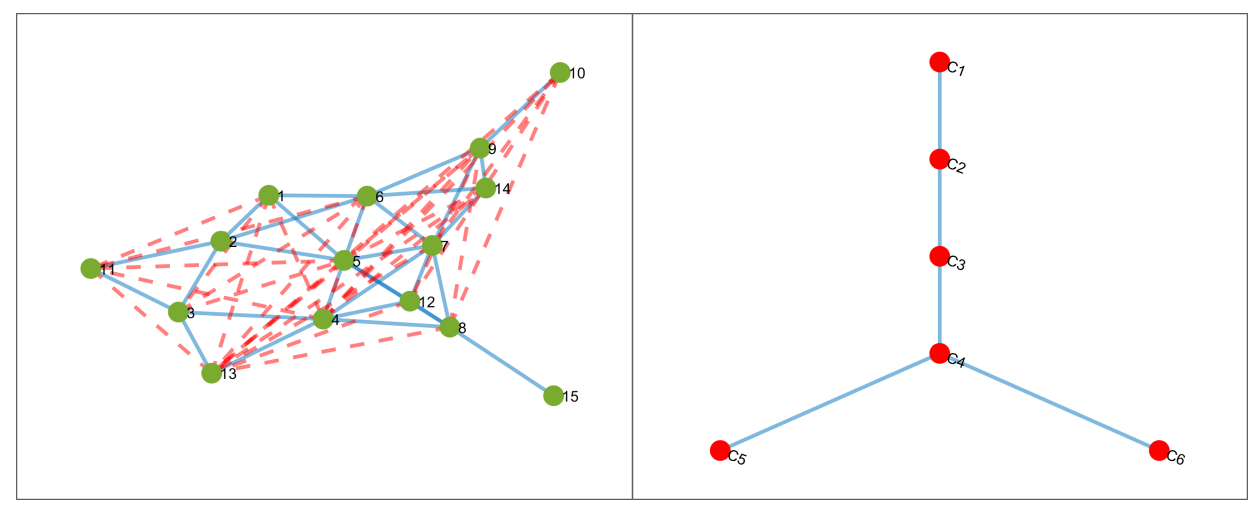

(a) Chordal embedding

(b) Clique tree

Figure 1.4: Second chordal embedding of the sparsity graph in Figure 1.1(b), and its corresponding clique tree

pointed out that an issue with using a sparsity graphs occurs when different terms of the optimization problem are assigned to the cliques. To be specific, checking the condition $J_{i} \subseteq C_{j}$ can be computationally demanding when the size of the sets $J_{i}$ and $C_{j}$ are large. This is not an issue when using a coupling graph. Notice that assigning terms to the clique tree in Figure 1.4(b) is more challenging than assigning terms to the clique tree in Figure 1.2(b). Also, when using a sparsity graph, the number of cliques in the resulting clique tree can be larger than the number of terms to be assigned. Therefore, in order to have a clique tree where all the cliques are assigned with optimization problem terms, the unassigned cliques should be merged with their neighboring cliques, which adds another task to the process of finding the computational graph. This is not the case when using a coupling graph, as the maximum number of the cliques in the resulting clique tree is bounded by the total number of the terms to be assigned. It should also be mentioned that using sparsity graphs can result in trees with a larger number of cliques compared to when using coupling graphs. In other words, given that a clique can be thought of as a computational agent, using sparsity graphs can potentially lead to algorithms which are more distributed in terms of computational agents used, than using coupling graphs. In this thesis, we use sparsity graphs in papers $\mathrm{A}$ and $\mathrm{B}$ and coupling graphs in papers $\mathrm{C}, \mathrm{D}$ and $\mathrm{E}$.

\subsubsection{Optimization over Clique Trees Using Message Passing}

Let us consider the optimization problem in $(1.1)$ and let $G(V, \mathcal{E})$ be the chordal embedding of the sparsity or coupling graph of this problem. Let $T\left(V_{t}, \mathcal{E}_{t}\right)$ be its corresponding clique tree with cliques $C_{Q}=\left\{C_{1}, \ldots, C_{q}\right\}$. Let us also assume that the terms in the equivalent problem in (1.4) are assigned based on the strategies discussed earlier, i.e. $\phi_{i}$ for $i=1, \ldots, q$ are selected. It is now possible to devise a distributed algorithm to solve the problem which uses the clique tree as its 
computational graph. The cliques in the clique tree act as computational agents which collectively solve the problem by exchanging messages through the edges of the clique tree. Next we express how the messages can be exchanged between cliques. Starting from the cliques in the leaf of the tree, each clique $i$ minimizes its assigned function with respect to the variables that are not shared with its parent clique $j$ and send the resulting function to its parent clique $j$, i.e.

$$
\begin{gathered}
m_{i j}\left(x_{A_{i j}}\right)=\min _{x_{B_{i j}}}\left\{\sum_{k \in \phi_{i}} \bar{F}_{k}\left(x_{J_{k}}\right)\right\}, \\
A_{i j}=\left\{\begin{array}{cl}
C_{i} \cap C_{j}, & \text { if } G(V, \mathcal{E}) \text { is a sparsity graph, } \\
\bigcup_{l \in C_{i} \cap C_{j}} J_{l}, & \text { if } G(V, \mathcal{E}) \text { is a coupling graph, } \\
B_{i j} \backslash\left(C_{i} \cap C_{j}\right), & \text { if } G(V, \mathcal{E}) \text { is a sparsity graph, } \\
\left\{\bigcup_{l \in C_{i}} J_{l}\right\} \backslash\left\{\bigcup_{l \in\left(C_{i} \cap C_{j}\right)} J_{l}\right\}, & \text { if } G(V, \mathcal{E}) \text { is a coupling graph. }
\end{array}\right.
\end{gathered}
$$

Notice that $A_{i j}$ and $B_{i j}$ are calculated differently depending on if $G(V, \mathcal{E})$ is a sparsity graph or a coupling graph, as the vertices in a sparsity graph correspond to the indexes of the variable $x$, whereas the vertices in a coupling graph correspond to the indexes of $\bar{F}_{i}$. The vectors $x_{A_{i j}}, x_{B_{i j}}$ and $x_{J_{k}}$ include components of $x$ that correspond to the elements in sets $A_{i j}, B_{i j}$ and $J_{k}$, respectively. We denote by $m_{i j}\left(x_{C_{i} \cap C_{j}}\right)$, a message function. For example for the clique tree in Figure 1.2(b), which is obtained from the coupling graph of the problem, the leaf cliques are $C_{4}$ and $C_{5}$ and the messages these two cliques send to their parent cliques, are

$$
\begin{gathered}
m_{43}\left(x_{6}, x_{7}, x_{9}, x_{14}\right)=\min _{x_{10}}\left\{\bar{F}_{5}\left(x_{9}, x_{10}\right)\right\}, \\
m_{52}\left(x_{4}, x_{5}, x_{7}, x_{8}, x_{12}\right)=\min _{x_{15}}\left\{\bar{F}_{7}\left(x_{8}, x_{15}\right)\right\} .
\end{gathered}
$$

Once each clique $j$ receives the messages from all of its children, they are added to its assigned function. After that, the resulting function is minimized with respect to the variables that are not shared with its parent clique $p$ and the resulting message function is sent to its parent clique $p$ :

$$
\begin{aligned}
& m_{j p}\left(x_{A_{j p}}\right)=\min _{x_{B_{j p}}}\left\{\sum_{k \in \phi_{j}} \bar{F}_{k}\left(x_{J_{k}}\right)+\sum_{k \in \operatorname{chi}\left(C_{j}\right)} m_{k j}\left(x_{D_{k j}}\right)\right\}, \\
& D_{k j}=\left\{\begin{array}{cl}
C_{k} \cap C_{j}, & \text { if } G(V, \mathcal{E}) \text { is a sparsity graph, } \\
\bigcup_{l \in C_{k} \cap C_{j}} J_{l}, & \text { if } G(V, \mathcal{E}) \text { is a coupling graph. }
\end{array}\right.
\end{aligned}
$$

The vector $x_{D_{k j}}$ includes components of $x$ that correspond to the elements in set $D_{k j}$. Hence, for the clique tree in Figure 1.2(b), the message sent from $C_{3}$ to $C_{2}$ is 
$m_{32}\left(x_{1}, x_{2}, x_{4}, x_{5}, x_{6}, x_{7}, x_{8}, x_{12}\right)=\min _{x_{9}, x_{14}}\left\{\bar{F}_{6}\left(x_{6}, x_{7}, x_{9}, x_{14}\right)+m_{43}\left(x_{6}, x_{7}, x_{9}, x_{14}\right)\right\}$, and from $C_{2}$ to $C_{1}$ is

$$
\begin{aligned}
& m_{21}\left(x_{1}, x_{2}, x_{3}, x_{4}, x_{5}, x_{6}, x_{13}\right)=\min _{x_{7}, x_{8}, x_{12}}\left\{\bar{F}_{3}\left(x_{4}, x_{5}, x_{7}, x_{8}, x_{12}\right)\right. \\
& \left.\quad+m_{52}\left(x_{4}, x_{5}, x_{7}, x_{8}, x_{12}\right)+m_{32}\left(x_{1}, x_{2}, x_{4}, x_{5}, x_{6}, x_{7}, x_{8}, x_{12}\right)\right\} .
\end{aligned}
$$

Finally, when the root clique $r$ receives the messages from all its children, its optimal solution can be computed by solving the following optimization problem

$$
\begin{gathered}
x_{A_{r r}}^{*}=\underset{x_{A_{r r}}}{\operatorname{argmin}}\left\{\sum_{k \in \phi_{r}} \bar{F}_{k}\left(x_{J_{k}}\right)+\sum_{k \in \operatorname{chi}\left(C_{r}\right)} m_{k r}\left(x_{D_{k r}}\right)\right\}, \\
A_{r r}=\left\{\begin{array}{cl}
C_{r}, & \text { if } G(V, \mathcal{E}) \text { is a sparsity graph, } \\
\bigcup_{l \in C_{r}} J_{l}, & \text { if } G(V, \mathcal{E}) \text { is a coupling graph. }
\end{array}\right.
\end{gathered}
$$

Therefore, for the clique tree in Figure 1.2(b):

$$
\begin{aligned}
& \left(x_{1}^{*}, x_{2}^{*}, x_{3}^{*}, x_{4}^{*}, x_{5}^{*}, x_{6}^{*}, x_{11}^{*}, x_{13}^{*}\right)=\underset{x_{1}, x_{2}, x_{3}, x_{4}, x_{5}, x_{6}, x_{11}, x_{13}}{\operatorname{argmin}}\left\{\bar{F}_{1}\left(x_{1}, x_{2}, x_{5}, x_{6}\right)\right. \\
& \left.\quad+\bar{F}_{2}\left(x_{2}, x_{3}, x_{11}\right)+\bar{F}_{4}\left(x_{3}, x_{4}, x_{13}\right)+m_{21}\left(x_{1}, x_{2}, x_{3}, x_{4}, x_{5}, x_{6}, x_{13}\right)\right\} .
\end{aligned}
$$

The root clique $r$ then sends its optimal solution to its children cliques and therefore, the children cliques can also compute their optimal solution as

$$
x_{B_{i r}}^{*}=\left.\underset{x_{B_{i r}}}{\operatorname{argmin}}\left\{\sum_{k \in \phi_{i}} \bar{F}_{k}\left(x_{J_{k}}\right)+\sum_{k \in \operatorname{chi}\left(C_{i}\right)} m_{k i}\left(x_{D_{k i}}\right)\right\}\right|_{x_{A_{r r}}^{*}} .
$$

Therefore, for the clique tree in Figure 1.2(b):

$$
\begin{aligned}
\left(x_{7}^{*}, x_{8}^{*}, x_{12}^{*}\right)=\underset{x_{7}, x_{8}, x_{12}}{\operatorname{argmin}}\{ & \bar{F}_{3}\left(x_{4}^{*}, x_{5}^{*}, x_{7}, x_{8}, x_{12}\right)+m_{52}\left(x_{4}^{*}, x_{5}^{*}, x_{7}, x_{8}, x_{12}\right) \\
& \left.+m_{32}\left(x_{1}^{*}, x_{2}^{*}, x_{4}^{*}, x_{5}^{*}, x_{6}^{*}, x_{7}, x_{8}, x_{12}\right)\right\} .
\end{aligned}
$$

Similarly the other cliques can compute their optimal solution by receiving the optimal solution from their parents. Therefore, the cliques $C_{3}, C_{4}$ and $C_{5}$ in Figure 1.2(b) find their solutions as

$$
\left(x_{9}^{*}, x_{14}^{*}\right)=\underset{x_{9}, x_{14}}{\operatorname{argmin}}\left\{\bar{F}_{6}\left(x_{6}^{*}, x_{7}^{*}, x_{9}, x_{14}\right)+m_{43}\left(x_{6}^{*}, x_{7}^{*}, x_{9}, x_{14}\right)\right\},
$$




$$
\begin{aligned}
& x_{10}^{*}=\underset{x_{10}}{\operatorname{argmin}}\left\{\bar{F}_{5}\left(x_{9}^{*}, x_{10}\right)\right\}, \\
& x_{15}^{*}=\underset{x_{15}}{\operatorname{argmin}}\left\{\bar{F}_{7}\left(x_{8}^{*}, x_{15}\right)\right\} .
\end{aligned}
$$

respectively. In summary, by the end of an upward-downward pass through the clique tree, all the cliques compute their optimal solution. It can be shown that the obtained solution is an optimal solution, see (Koller and Friedman, 2009, Ch. 10), for the proof. It should be pointed out that message passing over clique tree is closely related to non-serial dynamical programming. Non-serial dynamical programming is used for solving problems with general coupling structures, which was introduced in Bertele and Brioschi (1973). For related works see e.g., Koller and Friedman (2009); Moallemi (2007); Wainwright et al. (2005) and the references therein. Message passing can also be used within optimization algorithms to distribute the computations of search directions. This is closely connected to multi-frontal factorization techniques for factorization of matrices, see e.g., Khoshfetrat Pakazad et al. (2017). Moreover, all other computations in an optimization algorithm, including step size and the termination criterion, can often also be distributed over the clique tree. This property is extensively used in developing various distributed optimization algorithms in this thesis. 


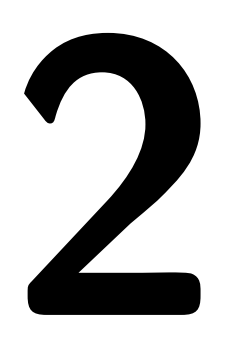

\section{Concluding Remarks and Future Work}

Rapid advances in technology in recent years have limited the use of centralized optimization approaches for large-scale complex systems due to the computational or communication challenges and/or structural constraints, which were discussed in the introduction. Therefore, the main objective of this thesis was to develop distributed optimization approaches, tailored for various applications in control and estimation by exploiting the inherent structures of the problems. To this end, we made use of chordal graphs, which enabled us to carry out the computations of the optimization algorithms distributedly using message passing over the computational graph of the problem.

In particular, we implemented a distributed primal-dual interior-point algorithm for loosely coupled QP problems and we discussed how parallelism can be exploited. We applied the algorithm on a linear MPC problem and we showed how one can benefit from parallel computations. Specifically, we showed how one can run the algorithm on multiple processors in parallel, in order to accelerate execution time of the algorithm compared to the case where the algorithm is run on a single processors. Also, we applied it on a robust MPC problem by converting the problem to an equivalent RQP formulation and we then showed how the chordal structure can be used to distribute the computations.

In addition, in this thesis, we presented a distributed optimization framework for a sensor network localization problem formulated with maximum likelihood estimation. The framework is based on the Levenberg-Marquardt algorithm in which the computations are carried out over the computational graph of the problem. We illustrated how the computational graph can be obtained distributedly. The resulting algorithm converged to an accurate solution in much fewer iterations than first-order methods, which led to fewer exchanged messages between computational agents. We also discussed the conditions in which the algorithm outperforms other algorithms in terms of root-mean-square error.

Moreover, we presented a distributed second-order augmented Lagrangian method 
for distributed MPC. In order to handle the inequality constraints, we adopted a primal-dual interior-point method in the inner iteration augmented Lagrangian method. We then showed how the computations of a centralized augmented Lagrangian method can be distributed using message passing over the computational graph of the problem. We illustrated the superiority of the algorithm in terms of number of iterations and communications compared to a distributed ADMM based method, using a set of numerical examples including a vehicle platooning problem.

Next, we point out a few directions for future research. The algorithms in papers A, B, D and E are based on Newton's method, meaning that the exact Hessian of the problem is used. When the Hessian is unavailable or is too expensive to compute, one can use a quasi-Newton method in which the Hessian is approximated with different formulas, such as Broyden-Fletcher-Goldfarb-Shanno (BFGS) or Broyden. Hence, one can investigate the possibility of distributing the computations of the approximate Hessian and use it instead of the exact Hessian.

In paper B, the obtained clique tree for the robust MPC problem has as many parallel branches as the number of what is called as scenarios in the paper. One possible extension is to introduce further parallelism for computations on each branch of the tree with the approach described in paper $\mathrm{A}$.

In paper $\mathrm{C}$, one can investigate different approaches for finding clique trees with parallel branches in order to facilitate parallel computations. In addition, different ways of initializing the algorithm in a cheap way can be explored.

In paper $\mathrm{E}$, it is required that at each iteration of the algorithm, the equality constraints are preprocessed by going an upward pass through the clique tree. This can be avoided by using a slightly different augmented Lagrangian function, defined as the generalized primal-dual augmented Lagrangian in Gill and Robinson (2012). The reason is that by using this function, the resulting Karush-KuhnTucker (KKT) system is guaranteed to be non-singular.

Moreover, in connection with paper E, an augmented Lagrangian interior-point approach is presented in Cao et al. (2016), where the linear KKT system is solved using a preconditioned conjugate gradient method, which is implemented efficiently on a Graphics Processing Unit (GPU) in parallel. For sufficiently sparse problems, one possible future work is to distribute the computations of the preconditioned conjugate gradient used for solving the KKT system, over the clique tree of the problem such that parallelism can be exploited. The results can then be compared with the ones in Cao et al. (2016). 


\section{Bibliography}

Shervin Parvini Ahmadi and Anders Hansson. Parallel exploitation for treestructured coupled quadratic programming in Julia. In 2018 22nd International Conference on System Theory, Control and Computing (ICSTCC), pages 597602. IEEE, 2018.

Shervin Parvini Ahmadi and Anders Hansson. A distributed second-order augmented Lagrangian method for distributed model predictive control. IFACPapersOnLine, 54(6):192-199, 2021a.

Shervin Parvini Ahmadi and Anders Hansson. Distributed model predictive control using an augmented Lagrangian method. Submitted for possible publication, 2021b.

Shervin Parvini Ahmadi, Anders Hansson, and Sina Khoshfetrat Pakazad. Efficient robust model predictive control using chordality. In 2019 18th European Control Conference (ECC), pages 4270-4275. IEEE, 2019.

Shervin Parvini Ahmadi, Anders Hansson, and Sina Khoshfetrat Pakazad. Distributed localization using Levenberg-Marquardt algorithm. EURASIP Journal on Advances in Signal Processing, 2021(1):1-26, 2021.

Umberto Bertele and Francesco Brioschi. On non-serial dynamic programming. $J$. Comb. Theory, Ser. A, 14(2):137-148, 1973.

Dimitri P Bertsekas and John N Tsitsiklis. Parallel and distributed computation: Numerical methods, 1997.

Yougang Bian, Shengbo Eben Li, Wei Ren, Jianqiang Wang, Keqiang Li, and Henry X Liu. Cooperation of multiple connected vehicles at unsignalized intersections: Distributed observation, optimization, and control. IEEE Transactions on Industrial Electronics, 67(12):10744-10754, 2019.

Pratik Biswas, T-C Liang, K-C Toh, Yinyu Ye, and T-C Wang. Semidefinite programming approaches for sensor network localization with noisy distance measurements. IEEE transactions on automation science and engineering, 3(4): 360-371, 2006. 
Jean RS Blair and Barry Peyton. An introduction to chordal graphs and clique trees. In Graph theory and sparse matrix computation, pages 1-29. Springer, USA, 1993.

Stephen Boyd, Neal Parikh, and Eric Chu. Distributed optimization and statistical learning via the alternating direction method of multipliers. Now Publishers Inc, 2011.

Francesco Bullo, Jorge Cortés, and Sonia Martínez. Distributed control of robotic networks: a mathematical approach to motion coordination algorithms. Technical report, PRINCETON UNIV NJ, 2008.

Yankai Cao, Arpan Seth, and Carl D Laird. An augmented Lagrangian interiorpoint approach for large-scale NLP problems on graphics processing units. Computers \& Chemical Engineering, 85:76-83, 2016.

Yongcan Cao, Wenwu Yu, Wei Ren, and Guanrong Chen. An overview of recent progress in the study of distributed multi-agent coordination. IEEE Transactions on Industrial informatics, 9(1):427-438, 2012.

Gong Chen and Marc Teboulle. A proximal-based decomposition method for convex minimization problems. Mathematical Programming, 64(1):81-101, 1994.

Benoît Colson and Philippe L Toint. Optimizing partially separable functions without derivatives. Optimization methods and software, 20(4-5):493-508, 2005.

Andrew R Conn, Nicholas IM Gould, and Philippe L Toint. Trust region methods. SIAM, 2000.

AR Conn, NIM Gould, and Ph L Toint LANCELOT. A Fortran package for large-scale nonlinear optimization (Release A). Springer Ser. Comput. Math, $17,1992$.

Quoc Tran Dinh, Ion Necoara, Carlo Savorgnan, and Moritz Diehl. An inexact perturbed path-following method for Lagrangian decomposition in large-scale separable convex optimization. SIAM Journal on Optimization, 23(1):95-125, 2013.

Jonathan Eckstein. Splitting methods for monotone operators with applications to parallel optimization. PhD thesis, Massachusetts Institute of Technology, 1989.

Ferdinando Fioretto, Enrico Pontelli, and William Yeoh. Distributed constraint optimization problems and applications: A survey. Journal of Artificial Intelligence Research, 61:623-698, 2018.

Michael Garstka, Mark Cannon, and Paul Goulart. COSMO: A conic operator splitting method for convex conic problems. Journal of Optimization Theory and Applications, 190(3):779-810, 2021.

Philip E Gill and Daniel P Robinson. A primal-dual augmented Lagrangian. Computational Optimization and Applications, 51(1):1-25, 2012. 
Martin Charles Golumbic. Algorithmic graph theory and perfect graphs. Elsevier, 2004 .

Jacek Gondzio and Robert Sarkissian. Parallel interior-point solver for structured linear programs. Mathematical Programming, 96(3):561-584, 2003.

Andreas Griewank and $\mathrm{Ph}$ L Toint. Local convergence analysis for partitioned quasi-Newton updates. Numerische Mathematik, 39(3):429-448, 1982a.

Andreas Griewank and $\mathrm{Ph} \mathrm{L}$ Toint. Partitioned variable metric updates for large structured optimization problems. Numerische Mathematik, 39(1):119137, $1982 \mathrm{~b}$.

Boris Houska, Janick Frasch, and Moritz Diehl. An augmented Lagrangian based algorithm for distributed nonconvex optimization. SIAM Journal on Optimization, 26(2):1101-1127, 2016.

Björn Johansson. On distributed optimization in networked systems. PhD thesis, KTH, 2008.

Amin Kargarian, Javad Mohammadi, Junyao Guo, Sambuddha Chakrabarti, Masoud Barati, Gabriela Hug, Soummya Kar, and Ross Baldick. Toward distributed/decentralized DC optimal power flow implementation in future electric power systems. IEEE Transactions on Smart Grid, 9(4):2574-2594, 2016.

Mohammad Khayatian, Mohammadreza Mehrabian, Edward Andert, Rachel Dedinsky, Sarthake Choudhary, Yingyan Lou, and Aviral Shirvastava. A survey on intersection management of connected autonomous vehicles. ACM Transactions on Cyber-Physical Systems, 4(4):1-27, 2020.

Sina Khoshfetrat Pakazad, Anders Hansson, Martin S Andersen, and Isak Nielsen. Distributed primal-dual interior-point methods for solving tree-structured coupled convex problems using message-passing. Optimization Methods and Software, 32(3):401-435, 2017.

Masakazu Kojima, Nimrod Megiddo, Shinji Mizuno, and Susumu Shindoh. Horizontal and vertical decomposition in interior point methods for linear programs. Citeseer, 1994.

Daphne Koller and Nir Friedman. Probabilistic graphical models: principles and techniques. MIT press, 2009.

Jean-Yves L'Excellent. Multifrontal methods: parallelism, memory usage and numerical aspects. PhD thesis, Ecole normale supérieure de lyon-ENS LYON, 2012 .

Andreas A Malikopoulos, Christos G Cassandras, and Yue J Zhang. A decentralized energy-optimal control framework for connected automated vehicles at signal-free intersections. Automatica, 93:244-256, 2018. 
Nico Meyer-Huebner, Michael Suriyah, and Thomas Leibfried. Distributed optimal power flow in hybrid AC-DC grids. IEEE Transactions on Power Systems, 34 (4):2937-2946, 2019.

Ciamac Cyrus Moallemi. A message-passing paradigm for optimization, volume 68. 2007.

Daniel K Molzahn, Florian Dörfler, Henrik Sandberg, Steven H Low, Sambuddha Chakrabarti, Ross Baldick, and Javad Lavaei. A survey of distributed optimization and control algorithms for electric power systems. IEEE Transactions on Smart Grid, 8(6):2941-2962, 2017.

I Necoara and JAK Suykens. Interior-point Lagrangian decomposition method for separable convex optimization. Journal of Optimization Theory and Applications, 143(3):567-588, 2009.

Angelia Nedic. Distributed gradient methods for convex machine learning problems in networks: Distributed optimization. IEEE Signal Processing Magazine, 37 (3):92-101, 2020.

Angelia Nedić and Ji Liu. Distributed optimization for control. Annual Review of Control, Robotics, and Autonomous Systems, 1:77-103, 2018.

Angelia Nedic and Asuman Ozdaglar. Distributed subgradient methods for multiagent optimization. IEEE Transactions on Automatic Control, 54(1):48-61, 2009 .

Angelia Nedić, Alex Olshevsky, and Michael G Rabbat. Network topology and communication-computation tradeoffs in decentralized optimization. Proceedings of the IEEE, 106(5):953-976, 2018.

Angelia Nedich et al. Convergence rate of distributed averaging dynamics and optimization in networks. Foundations and Trends® in Systems and Control, 2 (1):1-100, 2015.

Jorge Nocedal and Stephen Wright. Numerical optimization. Springer Science \& Business Media, 2006.

Sina Khoshfetrat Pakazad. Divide and conquer: Distributed optimization and robustness analysis. Department of Electrical Engineering, Linköping University, 2015.

Neal Parikh and Stephen Boyd. Block splitting for distributed optimization. Mathematical Programming Computation, 6(1):77-102, 2014.

Wei Ren and Yongcan Cao. Distributed coordination of multi-agent networks: emergent problems, models, and issues, volume 1. Springer, 2011.

Ali H Sayed. Adaptation, learning, and optimization over networks. Foundations and Trends in Machine Learning, 7(ARTICLE):311-801, 2014. 
Andrea Simonetto and Geert Leus. Distributed maximum likelihood sensor network localization. IEEE Transactions on Signal Processing, 62(6):1424-1437, 2014 .

Jonathan E Spingarn. Applications of the method of partial inverses to convex programming: decomposition. Mathematical Programming, 32(2):199-223, 1985.

Mehrdad Tajalli and Ali Hajbabaie. Distributed optimization and coordination algorithms for dynamic speed optimization of connected and autonomous vehicles in urban street networks. Transportation research part C: emerging technologies, 95:497-515, 2018.

Paul Tseng. Applications of a splitting algorithm to decomposition in convex programming and variational inequalities. SIAM Journal on Control and Optimization, 29(1):119-138, 1991.

Paul Tseng. Dual coordinate ascent methods for non-strictly convex minimization. Mathematical programming, 59(1):231-247, 1993.

John Nikolas Tsitsiklis. Problems in decentralized decision making and computation. Technical report, Massachusetts Inst of Tech Cambridge Lab for Information and Decision Systems, 1984.

Lieven Vandenberghe and Martin S Andersen. Chordal graphs and semidefinite optimization. Foundations and Trends in Optimization, 1(4):241-433, 2015.

Martin J Wainwright and Michael Irwin Jordan. Graphical models, exponential families, and variational inference. Now Publishers Inc, 2008.

Martin J Wainwright, Tommi S Jaakkola, and Alan S Willsky. MAP estimation via agreement on trees: message-passing and linear programming. IEEE transactions on information theory, 51(11):3697-3717, 2005.

Zizhuo Wang, Song Zheng, Yinyu Ye, and Stephen Boyd. Further relaxations of the semidefinite programming approach to sensor network localization. SIAM Journal on Optimization, 19(2):655-673, 2008.

Xianchao Xie. A recursive method to learn Bayesian network, 2020. URL https://se.mathworks.com/matlabcentral/fileexchange/ 20678-a-recursive-method-to-learn-bayesian-networkl.

Bo Yang and Mikael Johansson. Distributed optimization and games: A tutorial overview. Networked Control Systems, pages 109-148, 2010.

Tao Yang, Xinlei Yi, Junfeng Wu, Ye Yuan, Di Wu, Ziyang Meng, Yiguang Hong, Hong Wang, Zongli Lin, and Karl H Johansson. A survey of distributed optimization. Annual Reviews in Control, 47:278-305, 2019.

Yang Zheng. Chordal sparsity in control and optimization of large-scale systems. PhD thesis, University of Oxford, 2019. 
Yang Zheng, Richard P Mason, and Antonis Papachristodoulou. Scalable design of structured controllers using chordal decomposition. IEEE Transactions on Automatic Control, 63(3):752-767, 2017.

Yang Zheng, Maryam Kamgarpour, Aivar Sootla, and Antonis Papachristodoulou. Scalable analysis of linear networked systems via chordal decomposition. In 2018 European Control Conference (ECC), pages 2260-2265. IEEE, 2018.

Yang Zheng, Maryam Kamgarpour, Aivar Sootla, and Antonis Papachristodoulou. Distributed design for decentralized control using chordal decomposition and ADMM. IEEE Transactions on Control of Network Systems, 7(2):614-626, 2019.

Minghui Zhu and Sonia Martínez. Distributed optimization-based control of multiagent networks in complex environments. Springer, 2015. 
Part II

Publications 



\section{Papers}

The papers associated with this thesis have been removed for copyright reasons. For more details about these see:

https://doi.org/10.3384/9789179291983 


\section{PhD Dissertations \\ Division of Automatic Control \\ Linköping University}

M. Millnert: Identification and control of systems subject to abrupt changes. Thesis No. 82, 1982. ISBN 91-7372-542-0.

A. J. M. van Overbeek: On-line structure selection for the identification of multivariable systems. Thesis No. 86, 1982. ISBN 91-7372-586-2.

B. Bengtsson: On some control problems for queues. Thesis No. 87, 1982. ISBN 91-7372593-5.

S. Ljung: Fast algorithms for integral equations and least squares identification problems. Thesis No. 93, 1983. ISBN 91-7372-641-9.

H. Jonson: A Newton method for solving non-linear optimal control problems with general constraints. Thesis No. 104, 1983. ISBN 91-7372-718-0.

E. Trulsson: Adaptive control based on explicit criterion minimization. Thesis No. 106, 1983. ISBN 91-7372-728-8.

K. Nordström: Uncertainty, robustness and sensitivity reduction in the design of single input control systems. Thesis No. 162, 1987. ISBN 91-7870-170-8.

B. Wahlberg: On the identification and approximation of linear systems. Thesis No. 163, 1987. ISBN 91-7870-175-9.

S. Gunnarsson: Frequency domain aspects of modeling and control in adaptive systems. Thesis No. 194, 1988. ISBN 91-7870-380-8.

A. Isaksson: On system identification in one and two dimensions with signal processing applications. Thesis No. 196, 1988. ISBN 91-7870-383-2.

M. Viberg: Subspace fitting concepts in sensor array processing. Thesis No. 217, 1989. ISBN 91-7870-529-0.

K. Forsman: Constructive commutative algebra in nonlinear control theory. Thesis No. 261, 1991. ISBN 91-7870-827-3.

F. Gustafsson: Estimation of discrete parameters in linear systems. Thesis No. 271, 1992. ISBN 91-7870-876-1.

P. Nagy: Tools for knowledge-based signal processing with applications to system identification. Thesis No. 280, 1992. ISBN 91-7870-962-8.

T. Svensson: Mathematical tools and software for analysis and design of nonlinear control systems. Thesis No. 285, 1992. ISBN 91-7870-989-X.

S. Andersson: On dimension reduction in sensor array signal processing. Thesis No. 290, 1992. ISBN 91-7871-015-4.

H. Hjalmarsson: Aspects on incomplete modeling in system identification. Thesis No. 298, 1993. ISBN 91-7871-070-7.

I. Klein: Automatic synthesis of sequential control schemes. Thesis No. 305, 1993. ISBN 91-7871-090-1.

J.-E. Strömberg: A mode switching modelling philosophy. Thesis No. 353, 1994. ISBN 917871-430-3.

K. Wang Chen: Transformation and symbolic calculations in filtering and control. Thesis No. 361, 1994. ISBN 91-7871-467-2.

T. McKelvey: Identification of state-space models from time and frequency data. Thesis No. 380, 1995. ISBN 91-7871-531-8.

J. Sjöberg: Non-linear system identification with neural networks. Thesis No. 381, 1995. ISBN 91-7871-534-2.

R. Germundsson: Symbolic systems - theory, computation and applications. Thesis No. 389, 1995. ISBN 91-7871-578-4.

P. Pucar: Modeling and segmentation using multiple models. Thesis No. 405, 1995. ISBN 91-7871-627-6. 
H. Fortell: Algebraic approaches to normal forms and zero dynamics. Thesis No. 407, 1995. ISBN 91-7871-629-2.

A. Helmersson: Methods for robust gain scheduling. Thesis No. 406, 1995. ISBN 917871-628-4.

P. Lindskog: Methods, algorithms and tools for system identification based on prior knowledge. Thesis No. 436, 1996. ISBN 91-7871-424-8.

J. Gunnarsson: Symbolic methods and tools for discrete event dynamic systems. Thesis No. 477, 1997. ISBN 91-7871-917-8.

M. Jirstrand: Constructive methods for inequality constraints in control. Thesis No. 527, 1998. ISBN 91-7219-187-2.

U. Forssell: Closed-loop identification: Methods, theory, and applications. Thesis No. 566, 1999. ISBN 91-7219-432-4.

A. Stenman: Model on demand: Algorithms, analysis and applications. Thesis No. 571, 1999. ISBN 91-7219-450-2.

N. Bergman: Recursive Bayesian estimation: Navigation and tracking applications. Thesis No. 579, 1999. ISBN 91-7219-473-1.

K. Edström: Switched bond graphs: Simulation and analysis. Thesis No. 586, 1999. ISBN 91-7219-493-6.

M. Larsson: Behavioral and structural model based approaches to discrete diagnosis. Thesis No. 608, 1999. ISBN 91-7219-615-5.

F. Gunnarsson: Power control in cellular radio systems: Analysis, design and estimation. Thesis No. 623, 2000. ISBN 91-7219-689-0.

V. Einarsson: Model checking methods for mode switching systems. Thesis No. 652, 2000. ISBN 91-7219-836-2.

M. Norrlöf: Iterative learning control: Analysis, design, and experiments. Thesis No. 653, 2000. ISBN 91-7219-837-0.

F. Tjärnström: Variance expressions and model reduction in system identification. Thesis No. 730, 2002. ISBN 91-7373-253-2.

J. Löfberg: Minimax approaches to robust model predictive control. Thesis No. 812, 2003. ISBN 91-7373-622-8.

J. Roll: Local and piecewise affine approaches to system identification. Thesis No. 802, 2003. ISBN 91-7373-608-2.

J. Elbornsson: Analysis, estimation and compensation of mismatch effects in A/D converters. Thesis No. 811, 2003. ISBN 91-7373-621-X.

O. Härkegård: Backstepping and control allocation with applications to flight control. Thesis No. 820, 2003. ISBN 91-7373-647-3.

R. Wallin: Optimization algorithms for system analysis and identification. Thesis No. 919, 2004. ISBN 91-85297-19-4.

D. Lindgren: Projection methods for classification and identification. Thesis No. 915, 2005. ISBN 91-85297-06-2.

R. Karlsson: Particle Filtering for Positioning and Tracking Applications. Thesis No. 924, 2005. ISBN 91-85297-34-8.

J. Jansson: Collision Avoidance Theory with Applications to Automotive Collision Mitigation. Thesis No. 950, 2005. ISBN 91-85299-45-6.

E. Geijer Lundin: Uplink Load in CDMA Cellular Radio Systems. Thesis No. 977, 2005. ISBN 91-85457-49-3.

M. Enqvist: Linear Models of Nonlinear Systems. Thesis No. 985, 2005. ISBN 91-8545764-7.

T. B. Schön: Estimation of Nonlinear Dynamic Systems - Theory and Applications. Thesis No. 998, 2006. ISBN 91-85497-03-7.

I. Lind: Regressor and Structure Selection — Uses of ANOVA in System Identification. Thesis No. 1012, 2006. ISBN 91-85523-98-4. 
J. Gillberg: Frequency Domain Identification of Continuous-Time Systems Reconstruction and Robustness. Thesis No. 1031, 2006. ISBN 91-85523-34-8.

M. Gerdin: Identification and Estimation for Models Described by Differential-Algebraic Equations. Thesis No. 1046, 2006. ISBN 91-85643-87-4.

C. Grönwall: Ground Object Recognition using Laser Radar Data - Geometric Fitting, Performance Analysis, and Applications. Thesis No. 1055, 2006. ISBN 91-85643-53-X.

A. Eidehall: Tracking and threat assessment for automotive collision avoidance. Thesis No. 1066, 2007. ISBN 91-85643-10-6.

F. Eng: Non-Uniform Sampling in Statistical Signal Processing. Thesis No. 1082, 2007. ISBN 978-91-85715-49-7.

E. Wernholt: Multivariable Frequency-Domain Identification of Industrial Robots. Thesis No. 1138, 2007. ISBN 978-91-85895-72-4.

D. Axehill: Integer Quadratic Programming for Control and Communication. Thesis No. 1158, 2008. ISBN 978-91-85523-03-0.

G. Hendeby: Performance and Implementation Aspects of Nonlinear Filtering. Thesis No. 1161, 2008. ISBN 978-91-7393-979-9.

J. Sjöberg: Optimal Control and Model Reduction of Nonlinear DAE Models. Thesis No. 1166, 2008. ISBN 978-91-7393-964-5.

D. Törnqvist: Estimation and Detection with Applications to Navigation. Thesis No. 1216, 2008. ISBN 978-91-7393-785-6.

P-J. Nordlund: Efficient Estimation and Detection Methods for Airborne Applications. Thesis No. 1231, 2008. ISBN 978-91-7393-720-7.

H. Tidefelt: Differential-algebraic equations and matrix-valued singular perturbation. Thesis No. 1292, 2009. ISBN 978-91-7393-479-4.

H. Ohlsson: Regularization for Sparseness and Smoothness - Applications in System Identification and Signal Processing. Thesis No. 1351, 2010. ISBN 978-91-7393-287-5.

S. Moberg: Modeling and Control of Flexible Manipulators. Thesis No. 1349, 2010. ISBN 978-91-7393-289-9.

J. Wallén: Estimation-based iterative learning control. Thesis No. 1358, 2011. ISBN 97891-7393-255-4.

J. D. Hol: Sensor Fusion and Calibration of Inertial Sensors, Vision, Ultra-Wideband and GPS. Thesis No. 1368, 2011. ISBN 978-91-7393-197-7.

D. Ankelhed: On the Design of Low Order H-infinity Controllers. Thesis No. 1371, 2011. ISBN 978-91-7393-157-1.

C. Lundquist: Sensor Fusion for Automotive Applications. Thesis No. 1409, 2011. ISBN 978-91-7393-023-9.

P. Skoglar: Tracking and Planning for Surveillance Applications. Thesis No. 1432, 2012. ISBN 978-91-7519-941-2.

K. Granström: Extended target tracking using PHD filters. Thesis No. 1476, 2012. ISBN 978-91-7519-796-8.

C. Lyzell: Structural Reformulations in System Identification. Thesis No. 1475, 2012. ISBN 978-91-7519-800-2.

J. Callmer: Autonomous Localization in Unknown Environments. Thesis No. 1520, 2013. ISBN 978-91-7519-620-6.

D. Petersson: A Nonlinear Optimization Approach to H2-Optimal Modeling and Control. Thesis No. 1528, 2013. ISBN 978-91-7519-567-4.

Z. Sjanic: Navigation and Mapping for Aerial Vehicles Based on Inertial and Imaging Sensors. Thesis No. 1533, 2013. ISBN 978-91-7519-553-7.

F. Lindsten: Particle Filters and Markov Chains for Learning of Dynamical Systems. Thesis No. 1530, 2013. ISBN 978-91-7519-559-9. 
P. Axelsson: Sensor Fusion and Control Applied to Industrial Manipulators. Thesis No. 1585, 2014. ISBN 978-91-7519-368-7.

A. Carvalho Bittencourt: Modeling and Diagnosis of Friction and Wear in Industrial Robots. Thesis No. 1617, 2014. ISBN 978-91-7519-251-2.

M. Skoglund: Inertial Navigation and Mapping for Autonomous Vehicles. Thesis No. 1623, 2014. ISBN 978-91-7519-233-8.

S. Khoshfetrat Pakazad: Divide and Conquer: Distributed Optimization and Robustness Analysis. Thesis No. 1676, 2015. ISBN 978-91-7519-050-1.

T. Ardeshiri: Analytical Approximations for Bayesian Inference. Thesis No. 1710, 2015. ISBN 978-91-7685-930-8.

N. Wahlström: Modeling of Magnetic Fields and Extended Objects for Localization Applications. Thesis No. 1723, 2015. ISBN 978-91-7685-903-2.

J. Dahlin: Accelerating Monte Carlo methods for Bayesian inference in dynamical models. Thesis No. 1754, 2016. ISBN 978-91-7685-797-7.

M. Kok: Probabilistic modeling for sensor fusion with inertial measurements. Thesis No. 1814, 2016. ISBN 978-91-7685-621-5.

J. Linder: Indirect System Identification for Unknown Input Problems: With Applications to Ships. Thesis No. 1829, 2017. ISBN 978-91-7685-588-1.

M. Roth: Advanced Kalman Filtering Approaches to Bayesian State Estimation. Thesis No. 1832, 2017. ISBN 978-91-7685-578-2.

I. Nielsen: Structure-Exploiting Numerical Algorithms for Optimal Control. Thesis No. 1848, 2017. ISBN 978-91-7685-528-7.

D. Simon: Fighter Aircraft Maneuver Limiting Using MPC: Theory and Application. Thesis No. 1881, 2017. ISBN 978-91-7685-450-1.

C. Veibäck: Tracking the Wanders of Nature. Thesis No. 1958, 2018. ISBN 978-91-7685200-2.

C. Andersson Naesseth: Machine learning using approximate inference: Variational and sequential Monte Carlo methods. Thesis No. 1969, 2018. ISBN 978-91-7685-161-6.

Y. Jung: Inverse system identification with applications in predistortion. Thesis No. 1966, 2018. ISBN 978-91-7685-171-5.

Y. Zhao: Gaussian Processes for Positioning Using Radio Signal Strength Measurements. Thesis No. 1968, 2019. ISBN 978-91-7685-162-3.

R. Larsson: Flight Test System Identification. Thesis No. 1990, 2019. ISBN 978-91-7685070-1.

P. Kasebzadeh: Learning Human Gait. Thesis No. 2012, 2019. ISBN 978-91-7519-014-3.

K. Radnosrati: Time of flight estimation for radio network positioning. Thesis No. 2054, 2020. ISBN 978-91-7929-884-5.

O. Ljungqvist: Motion planning and feedback control techniques with applications to long tractor-trailer vehicles. Thesis No. 2070, 2020. ISBN 978-91-7929-858-6.

G. Lindmark: Controllability of Complex Networks at Minimum Cost. Thesis No. 2074, 2020. ISBN 978-91-7929-847-0.

K. Bergman: Exploiting Direct Optimal Control for Motion Planning in Unstructured Environments. Thesis No. 2133, 2021. ISBN 978-91-7929-677-3.

P. Boström-Rost: Sensor Management for Target Tracking Applications. Thesis No. 2137, 2021. ISBN 978-91-7929-672-8.

A. Fontan: Collective decision-making on networked systems in presence of antagonistic interactions. Thesis No. 2166, 2021. ISBN 978-91-7929-017-7. 



\section{FACULTY OF SCIENCE AND ENGINEERING}

Linköping Studies in Science and Technology, Dissertation No. 2207, 2022 Department of Electrical Engineering

Linköping University

SE-581 83 Linköping, Sweden

www.liu.se 